\title{
Potential of Parametric X-rays for Application in Particle Identification Detectors ${ }^{\dagger}$
}

\author{
Alexander S. Lobko *, Victor V. Tikhomirov and Alesia V. Leukovich \\ Institute for Nuclear Problems, Belarusian State University, Bobrujskaya Str., Bdg 11, 220006 Minsk, Belarus \\ * Correspondence: alexander.lobko@cern.ch \\ + Presented at the 37th International Symposium on Dynamical Properties of Solids (DyProSo 2019), Ferrara, \\ Italy, 8-12 September 2019.
}

Published: 5 September 2019

Cerenkov counters and transition radiation detectors are particle identification detectors (PID) used in many HEP experiments [1,2]. Study and development of new accelerator facilities such as Future Circular Colliders (CERN), NICA (JINR) and others formulate new needs for particle identification in conditions of very high energies and luminosities. Parametric X-rays (PXR) [3] have big potential to be applied in particle identification detectors as radiation mechanism combining many properties of Cerenkov radiation and transition radiation in high kinematics range $\gamma>1000$. PXR mechanism well developed to date, both theoretically and experimentally. Many experiments were performed with electron beams. Key experiment with first observation of PXR from $70 \mathrm{GeV}$ protons was reported in [4]. Later, PXR has been studied with $5 \mathrm{GeV}$ protons and $2.2 \mathrm{GeV} / \mathrm{u}$ carbon nuclei in a silicon crystal on the external beams of the Nuclotron at JINR [5] and with $400 \mathrm{GeV} / \mathrm{c}$ protons in bent crystals at CERN SPS [6].

So, we may state, that the community reached good understanding of the PXR properties by now and it is ready for the feasibility study of PXR application in particle identification. In contribution we will present and discuss characteristic features of PXR potentially applicable for PID including time dependences as timing requirements are of high importance for future colliders due to their increased collision rates. As first step for PXR application for high energy particle detection, implementation of PXR simulation tool to the GEANT4 toolkit can be proposed.

\section{References}

1. Krizan, P. Detectors for particle identification. Nucl. Instrum. Methods A 2007, 581, 57-64.

2. Andronic, A.; Wessels, J.P. Transition radiation detectors. Nucl. Instrum. Methods A 2012, 666, $130-147$.

3. Baryshevsky, V.G.; Feranchuk, I.D.; Ulyanenkov, A.P. Parametric X-ray Radiation in Crystals: Theory, Experiments and Applications; Springer: Berlin/Heidelberg, Germany, 2005.

4. Afanasenko, V.P.; Baryshevskii, V.G.; Zuevskii, R.F.; Lobko, A.S.; Moskatel'nikov, A.A.; Panov, V.V.; Potsiluiko, V.P.; Skorokhod, S.V.; Shvarkov, D.S.; Parsons, D. Observation of parametric X-ray emission by protons. JETP Lett. 1991, 54, 494-497.

5. Adischev, Y.N.; Afanasiev, S.V.; Boiko, V.V.; Efimov, A.N.; Efremov, Y.V.; Gogolev, A.S.; Kovalenko, A.D.; Pivovarov, Y.L.; Potylitsyn, A.P.; Romanov, S.V.; et al. First observation of parametric X-rays produced by moderate relativistic protons and carbon nuclei in Si crystals. Nucl. Instrum. Methods B 2006, 252, 111-117.

6. Scandale, W.; Arduini, G.; Assmann, R.; Cerutti, F.; Gilardoni, S.; Christiansen, J.; Laface, E.; Losito, R.; Masi, A.; Metral, E.; et al. Observation of parametric X-rays produced by $400 \mathrm{GeV} / \mathrm{c}$ protons in bent crystals. Phys. Lett. B 2011, 701, 180-185. 